\title{
Computing Effective Hamiltonians of Coupled Electromagnetic Systems
}

This paper was downloaded from TechRxiv (https://www.techrxiv.org).

LICENSE

CC BY 4.0

SUBMISSION DATE / POSTED DATE

$25-08-2021$ / 26-08-2021

CITATION

Liao, Shaolin; Ou, Lu (2021): Computing Effective Hamiltonians of Coupled Electromagnetic Systems. TechRxiv. Preprint. https://doi.org/10.36227/techrxiv.16437300.v1

$\mathrm{DOI}$

10.36227/techrxiv.16437300.v1 


\title{
Computing Effective Hamiltonians of Coupled Electromagnetic Systems
}

\author{
Shaolin $\mathrm{Liao}^{\mathrm{a}}$ and $\mathrm{Lu} \mathrm{O \textrm {O } ^ { \mathrm { b } }}$

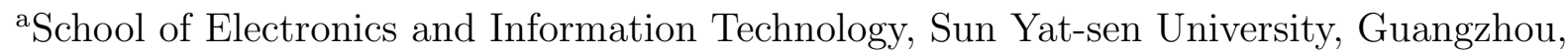 \\ Guangdong Province, China and Department of Electrical and Computer Engineering, Illinois \\ Institute of Technology, Chicago, IL, USA 60616 \\ ${ }^{\mathrm{b}}$ School of Journalism and Communication, Hunan University, Changsha, Hunan, China \\ 410082
}

\begin{abstract}
In this paper, we present an efficient procedure to compute the effective Hamiltonian matrix of a coupled electromagnetic system consisting of subsystems that are coupled to a discrete number of channels through couplers. Each subsystem is described by its own effective non-Hermitian Hamiltonian and the corresponding Quasi-normal Modes (QNMs), while the coupler connecting the subsystems and the channels is described by the scattering matrix, which is equivalent to the transfer matrix, in terms of port vectors defined for the coupler. Due to the constraints imposed by the QNMs of the subsystems and the wave dynamics of the channels, as well as boundary condition constraints, constraint-free port vectors need to be chosen efficiently and they follow two rules: 1) port vectors forming loops with couplers; 2) port vectors of couplers with most constraints or with less freedom. With the constraint-free port vectors chosen, the effective Hamiltonian matrix of the coupled electromagnetic system can be obtained by imposing the boundary condition constraints. After the effective Hamiltonian is obtained, the eigenvalues, eigenvectors and dispersion relation of the coupled electromagnetic system, as well as other quantities such as the reflection and transmission, can be calculated. A 2D interstitial square coupled MRRs array is used as an example to demonstrate the computational procedure. The computation of the effective Hamiltonian matrix of a coupled electromagnetic system has many potential applications such as MRRs array, coupled Parity-Time Non-Hermitian electromagnetic system, as well as the dispersion relation of finite and infinite arrays.
\end{abstract}

Keywords: Coupled electromagnetic system, effective Hamiltonian matrix, scattering matrix, transfer matrix, eigenmodes, eigenvalues, eigenvectors, reflection, transmission, microring resonators, arrays.

\section{INTRODUCTION}

Coupled electromagnetic systems are ubiquitous in real life, for example, the coupled photonics microring resonator filters, ${ }^{1}$ coupled Parity-Time Non-Hermitian electromagnetic system, ${ }^{2,3}$ as well as the finite and infinite coupled Microring Resonators (MRRs) array. ${ }^{4}$ For example, in the past decades, MRRs have been considered as one of the strong candidates for building blocks of the optical components and devices such as the high-performance filter such as add-drop filters ${ }^{5}$ and wavelength division multiplexers, ${ }^{6},{ }_{7}^{7}$ optical delay lines, ${ }^{8}$ Parity-Time (PT) symmetric devices, ${ }^{9}$ nonlinear light-wave and materials interaction such as four wave mixing, ${ }^{10}$ single-photon/photon-pair source, ${ }^{11},{ }^{12}$ frequency comb generation, ${ }^{13}$ optical quantum computing, ${ }^{14}$ as well as high-quality sensors. ${ }^{15}$ All of these call for efficient computation of the effective Hamiltonian matrix of the coupled electromagnetic system so that the optimal system can be designed for better performance, which is the focus of this paper.

Further author information: (Send correspondence to Shaolin Liao)

Shaolin Liao: E-mail: liaoshlin@mail.sysu.edu.cn, Telephone: +86 17680607708

Lu Ou: E-mail: oulu9676@gmail.com, Telephone: +86 18569525889 


\section{PROBLEM STATEMENT}

The coupled electromagnetic system is shown in Fig. 1: it consists of a total of $M$ subsystems that are coupled to each other through their mutual coupling channels:

First, subsystem $m$ is described by its own eigenmodes $\mathbf{E}_{i, m}, i=1,2, \cdots I_{m}$ that are governed by its Hamiltonian $\overline{\bar{H}}_{m}, m=1,2, \cdots, M$.

Then channel $n$ can be described in its forward-propagating wave basis $\mathbf{E}_{n}^{+}(\mathbf{r})$ and backward-propagating wave basis $\mathbf{E}_{n}^{-}(\mathbf{r})$ as $\bar{v}_{n}=\left[\mathbf{a}_{n}, \mathbf{b}_{n}\right]^{T}, n=1,2, \cdots, N$, with $\mathbf{a}_{n}$ and $\mathbf{b}_{n}$ being the complex coefficients of the forward-propagating wave basis $\mathbf{E}^{+}(\mathbf{r})$ and backward-propagating wave $\mathbf{E}^{-}(\mathbf{r})$ basis of channel $n$ respectively.

Finally, coupler $l$ connects subsystem $m$ and to some channels $n_{l}$, which can be described by the scattering matrix $\overline{\bar{S}}_{l}$, which is equivalent to the transfer matrix $\overline{\bar{T}}_{l}$.

The goal of this paper is to obtain the effective non-Hermitian Hamiltonian of the coupled electromagnetic system.

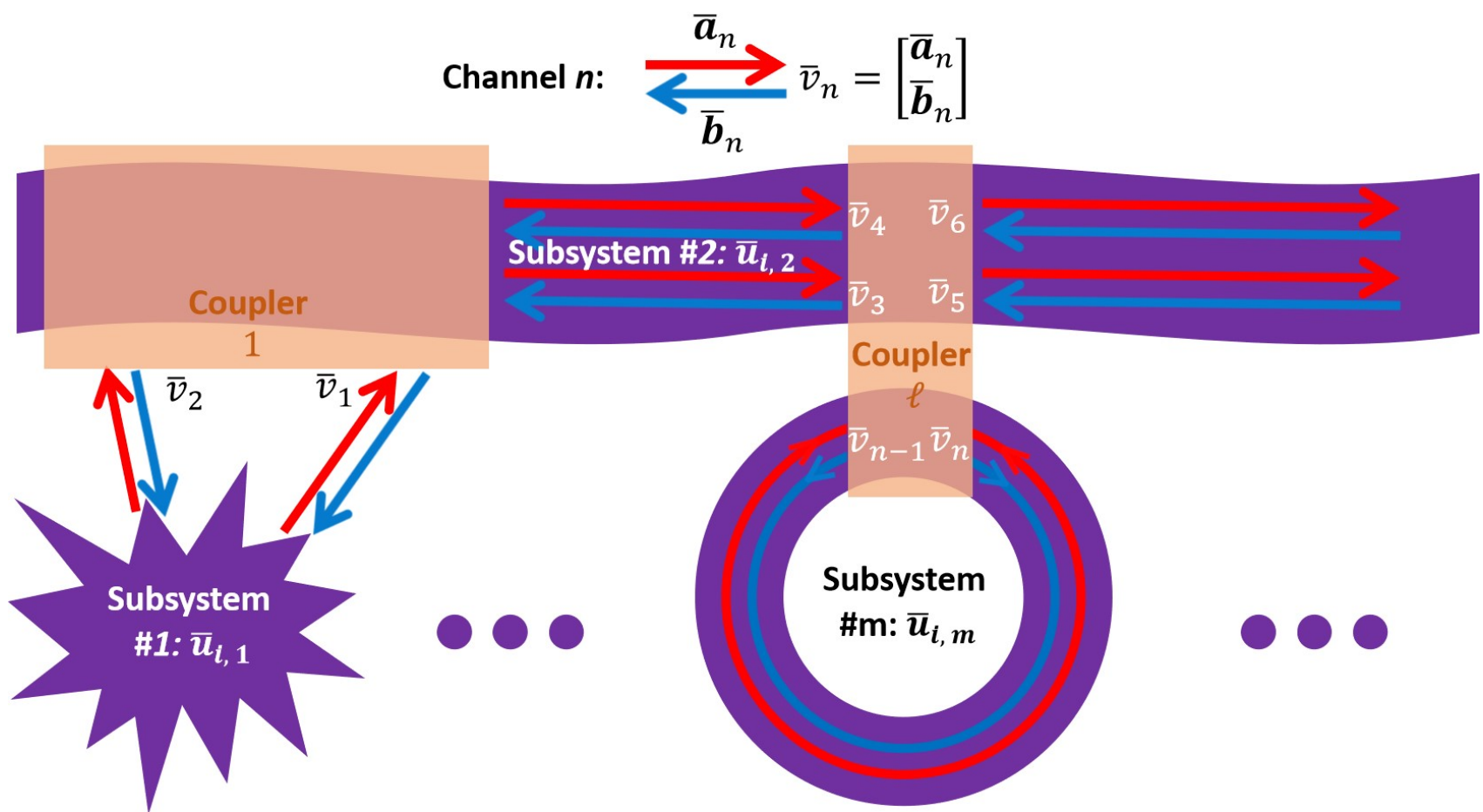

Figure 1: The coupled electromagnetic system consists of $M$ subsystems, which are coupled to each other through their mutual coupling channels $\bar{v}_{l, n}, n=1,2, \cdots N$.

\subsection{The Electromagnetic Subsystem}

The eigenmode $i$ of subsystem $m$ is a solution of the Maxwell's equations constrained by its boundary condition,

$$
\Delta \mathbf{E}_{i, m}(\mathbf{r})+k_{m}^{2}(\omega) \mathbf{E}_{i, m}(\mathbf{r})=0,
$$

where $k_{m}(\omega)$ is the magnitude of the wavevector at frequency $\omega$.

The eigenmode $\mathbf{E}_{i, m}(\mathbf{r})$ can be expressed as the sum of the forward-propagating wave $\mathbf{E}_{i, m}^{+}(\mathbf{r})$ and backwardpropagating wave $\mathbf{E}_{i, m}^{-}(\mathbf{r})$ in terms of the superposition of plane waves,

$$
\mathbf{E}_{i, m}(\mathbf{r})=\mathbf{E}_{i, m}^{+}(\mathbf{r})+\mathbf{E}_{i, m}^{-}(\mathbf{r}),
$$


with

$$
\mathbf{E}_{i, m}^{+}(\mathbf{r})=\int_{-\infty}^{\infty} \int_{-\infty}^{\infty} \alpha_{i, m}^{+}\left(\mathbf{k}_{\rho}\right) \exp ^{+j\left(\mathbf{k}_{\rho} \cdot \boldsymbol{\rho}+k_{z} z\right)} d \mathbf{k}_{\rho}, \quad \mathbf{E}_{i, m}^{-}(\mathbf{r})=\int_{-\infty}^{\infty} \int_{-\infty}^{\infty} \alpha_{i, m}^{-}\left(\mathbf{k}_{\rho}\right) \exp ^{-j\left(\mathbf{k}_{\rho} \cdot \boldsymbol{\rho}+k_{z} z\right)} d \mathbf{k}_{\rho} .
$$

From the Maxwell's equation in Eq. (1), if $\mathbf{E}_{i, m}^{+}(\mathbf{r})$ is a solution, then its conjugate $\mathbf{E}_{i, m}^{+*}(\mathbf{r})$ is also a solution, which leads to the following relation

$$
\mathbf{E}_{i, m}^{-}(\mathbf{r})=\xi_{i, m} \mathbf{E}_{i, m}^{+*}(\mathbf{r}) \rightarrow \alpha_{i, m}^{-}\left(\mathbf{k}_{\rho}\right)=\xi_{i, m} \alpha_{i, m}^{+*}\left(\mathbf{k}_{\rho}\right),
$$

from which the eigenmode $\mathbf{E}_{i, m}(\mathbf{r})$ can be expressed in the basis set of the forward-propagating wave $\mathbf{E}_{i, m}^{+}(\mathbf{r})$ and backward-propagating wave $\mathbf{E}_{i, m}^{-}(\mathbf{r})$ as follows,

$$
\bar{u}_{i, m}=c_{i, m}\left[1, \xi_{i, m}\right]^{T},
$$

where $\xi_{i, m}=1$ for Hermitian close subsystem and $\xi_{i, m} \sim 1$ for weak coupling to the external environment, which can be dealt as Quasi-normal Modes (QNMs).

\subsection{The Channels}

Similarly, channel $m$ consists of its forward-propagating $\mathbf{E}_{n}^{+}(\mathbf{r})$ and backward-propagating waves $\mathbf{E}_{n}^{-}(\mathbf{r})=\mathbf{E}_{\mathbf{n}}^{+*}(\mathbf{r})$. So, each channel can be described in terms of the forward-propagating wave $\mathbf{E}_{n}^{+}(\mathbf{r})$ and backward-propagating wave $\mathbf{E}_{n}^{+*}(\mathbf{r})$., along with their complex coefficients as follows,

$$
\mathbf{E}_{n}(\mathbf{r})=\mathbf{a}_{n} \mathbf{E}_{n}^{+}(\mathbf{r})+\mathbf{b}_{n} \mathbf{E}_{n}^{+*}(\mathbf{r}),
$$

which can be compactly expressed in the vector format as follows,

$$
\bar{v}_{n}=\left[a_{n}, b_{n}\right]^{T}, n=1,2, \cdots, N,
$$

where $N$ is the total number the channels.

It can be seen that the coefficients $\mathbf{a}_{n}$ and $\mathbf{b}_{n}$ of the channel vector in Eq. (7) are independent from each other, which is different from those of the eigenmode vector in Eq. (5)

\subsection{The Coupler}

A coupler $l$ that connects $L_{n}$ coupling channels to $L_{m}$ eigenmodes of subsystem $m$ is called a $L_{m} \times L_{n}$ coupler. The eigenmode vector and the coupling channel vector defined at the coupler are called the eigenmode port vector and the coupling channel port vector respectively. They are related to each other through the coupler's scattering matrix, which is equivalent to its transfer matrix as follows,

$$
\bar{u}_{m, l}=\overline{\bar{T}}_{m, l}^{L_{m} \times L_{n}} \bar{v}_{l},
$$

with

$$
\bar{u}_{l, m}=\left[\cdots, \bar{u}_{l, i, m}^{T}, \cdots\right]^{T}, \quad \bar{v}_{l}=\left[\cdots, \bar{v}_{l, n}^{T}, \cdots\right]^{T} .
$$

\subsection{Constraints on the Subsystems and Channels}

Furthermore, since the electromagnetic wave dynamic of each subsystem and channel is governed by their own Hamiltonians, the eigenmode port vectors and channel port vector at different couplers are related,

$$
\bar{u}_{l^{\prime}, i, m}=\overline{\bar{H}}_{l^{\prime}, l, i, m} \bar{u}_{l, i, m}, \quad \bar{v}_{l^{\prime}, n}=\overline{\bar{P}}_{l^{\prime}, l, n} \bar{v}_{l, n},
$$

where $\overline{\bar{H}}_{l^{\prime}, l, i, m}$ denotes the constraint on the eigenmode port vector pair $\left\{\left(l^{\prime}, i, m\right),(l, i, m)\right\}$ of the subsystem and $\overline{\bar{P}}_{l^{\prime}, l, n}$ denotes the constraint on the channel port vector pair $\left\{\left(l^{\prime}, n^{\prime}\right),(l, n)\right\}$ of the channel. 
From Eq. (3) and Eq. (5), $\overline{\bar{H}}_{l^{\prime}, l, i, m}$ of subsystem $m$ is obtained as follows,

$$
\overline{\bar{H}}_{l^{\prime}, l, i, m} \sim \frac{\mathbf{E}_{i, m}\left(\mathbf{r}_{l^{\prime}}\right)}{\mathbf{E}_{i, m}\left(\mathbf{r}_{l}\right)} \overline{\bar{I}} \sim \frac{\Re\left(\mathbf{E}_{i, m}^{+}\left(\mathbf{r}_{l^{\prime}}\right)\right)}{\Re\left(\mathbf{E}_{i, m}^{+}\left(\mathbf{r}_{l}\right)\right)} \overline{\bar{I}} .
$$

where $\overline{\bar{I}}$ is the identity matrix.

Similarly, From Eq. (8), $\overline{\bar{P}}_{l^{\prime}, l, n}$ of channel $n$ is obtained as follows,

$$
\overline{\bar{P}}_{l^{\prime}, l, n}=\left[\begin{array}{cc}
\exp ^{+j k_{n} d_{l^{\prime}, l}} & 0 \\
0 & \exp ^{-j k_{n} d_{l^{\prime}, l}}
\end{array}\right]
$$

where $k_{n}$ denotes the wave vector of channel $n$ and $d_{l^{\prime}, l}$ is the optics length from coupler $l$ to coupler $l^{\prime}$.

\subsection{Boundary Condition Constraints}

The boundary conditions apply another type of constraint on the port vectors. The boundary conditions include the coupling to the external system (e.g., with an external coupler) and the periodic Bloch-Floquent (BF) boundary condition for infinite array structures. In particular, the periodic Bloch-Floquent boundary condition for the array structure can be expressed as follows,

$$
\bar{v}_{B F}^{+}=\overline{\bar{P}}\{\mathbf{k}(\omega), \mathbf{p}\} \bar{v}_{B F}^{-},
$$

where denotes the periodic BF boundary condition constraint.

\subsection{Constraint-free Coupled System}

Due to the constraints of the subsystems and channels, as well as the boundary condition constraints, the total number of constraint-free port vectors has to be determined.

First, due the channel constraint, the total number of constraint-free channel port vectors in Eq. (8) reduces to $L_{n}^{\prime}=L_{n}-N_{l}$ if there are $N_{l}$ channels forming loops with coupler $l$; Similarly, the total number of constraintfree eigenmode port vectors reduces to $L_{m}^{\prime}=L_{m}-M_{l}$ if there is $M_{l}$ eigenmodes forming loops with coupler $l$. So the transfer matrix relation of Eq. (8) reduces to the newly formed transfer matrix with all the constraint-free port vectors

$$
\bar{u}_{m, l}^{\prime}=\overline{\bar{T}}_{m, l}^{L_{m}^{\prime} \times L_{n}^{\prime}} \bar{v}_{l}^{\prime}
$$

Then, supposing that there are total of $N_{B}$ equations due to the boundary condition constraints and $N_{B F}$ Bloch-Floquent boundary condition constraints, then the total number of constraints-free port vectors $L$ is given by

$$
L=\sum_{l}\left(L_{m}^{\prime}+L_{n}^{\prime}\right)-\left(N_{B}+N_{B F}\right)
$$

\section{RECIPE FOR COMPUTATION OF THE EFFECTIVE HAMILTONIAN}

Now, the $L$ constraint-free port vectors remain to be chosen so that the effective Hamiltonian can be computed, which is presented as follows,

1. First, the unknown port vectors related to the boundary condition constraints, i.e., the external boundary condition constraint and the Bloch-Floquent boundary condition constraint, are chosen as the constraintfree port vectors $\bar{v}_{B}=\bar{i}$. 
2. Second, it can be seen that both each eigenmode constraint or channel constraint forms loop with the $l$-th coupler and thus relates the two port vectors at the coupler. So it is efficient to choose one of the port vector $\bar{v}_{i, l, m}$ or $\bar{v}_{n, l}$ as the constraint-free port vector $\bar{v}_{l}^{c}$ so that the other can be obtained through the constraint relation given in Eq. (10). Supposing the total number of the chosen constraint-free port vectors $\bar{v}_{l}^{c}$ is $N_{l}^{c}$, there will be $N_{l}^{\prime}=N_{l}-N_{l}^{c}$ constraint-free port vectors left to be determined.

3. Then, the coupler that have the minimum constraint-free port vectors $\bar{v}_{l}^{c^{\prime}}$ will be chosen so that the coupler can be solved for all of its remaining port vectors.

4. Furthermore, Step 2. is repeated until all constraint-free port vectors are found and the remaining port vectors can be solved, together with the help of the $N_{o}$ constraint-free output port vectors $\bar{v}_{l}^{o}$.

5. Now, the effective Hamiltonian can be obtained as follows:

(a) For each coupler $l$, the right-side port vectors $\bar{v}_{l}^{+}$could be be obtained as follows,

$$
\bar{v}_{l}^{+}=\overline{\bar{T}}_{l} \bar{v}_{l}^{-} .
$$

The other way to obtain the right-side port vectors of coupler $\bar{v}_{l}^{+}$is through their coupling with other couplers,

$$
\bar{v}_{l}^{+}=\overline{\bar{T}}_{l^{\prime}} \bar{v}_{l^{\prime}}^{-}
$$

where $\bar{v}_{l^{\prime}}^{-}$denotes the left-side port vectors of coupler $l^{\prime}$ that are coupled to the right-side port vectors of coupler $l$ and $\overline{\bar{T}}_{l^{\prime}}$ denotes the coupling transfer matrix.

Now, a set of equations can be formed by equating Eq. (16) and Eq. (17),

$$
\overline{\bar{T}}_{l} \bar{v}_{l}=\overline{\bar{T}}_{l^{\prime}} \bar{v}_{l^{\prime}}
$$

(b) Repeat Step (a) until all equations that relate all constraint-free port vectors are obtained, including the boundary condition constraints, from which the effective Hamiltonian can be obtained as follows,

$$
\overline{\bar{H}}^{L \times L}(\omega) \bar{v}^{L}=\bar{v}^{i},
$$

where $\bar{v}^{i}$ is related to the input port vector.

(c) Finally, the coupled electromagnetic system can be solved because the total number of equations in Eq. (19) equals the total number of unknown constraint-free port vectors $\bar{v}^{L}$.

i. For the coupled electromagnetic system that is coupled to the external envoronment, Eq. (19) can be solved as follows,

$$
\bar{v}^{L}=\left[\overline{\bar{H}}^{L \times L}\right]^{-1} \bar{v}^{i} .
$$

ii. For standalone coupled electromagnetic system, $\bar{v}^{i}=0$, Eq. (19) reduces to the eigen problem,

$$
\overline{\bar{H}}^{L \times L}(\omega) \bar{v}^{L}=0,
$$

from which the eigen frequencies can be obtained,

$$
\text { Det } \equiv\left|\overline{\bar{H}}^{L \times L}(\omega)\right|=0 .
$$




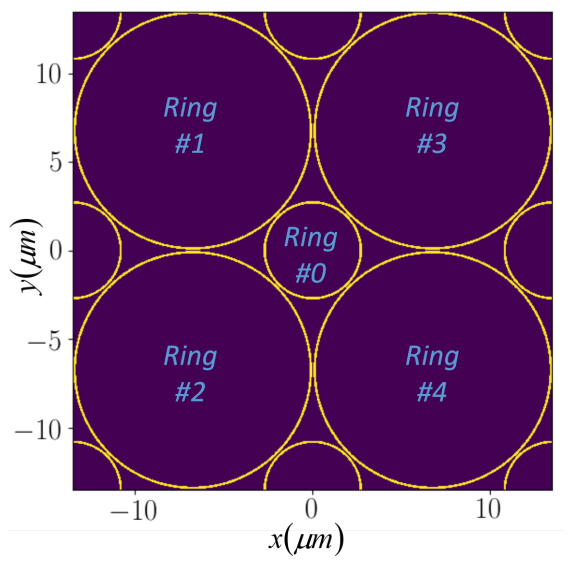

(a) Four unit cells.

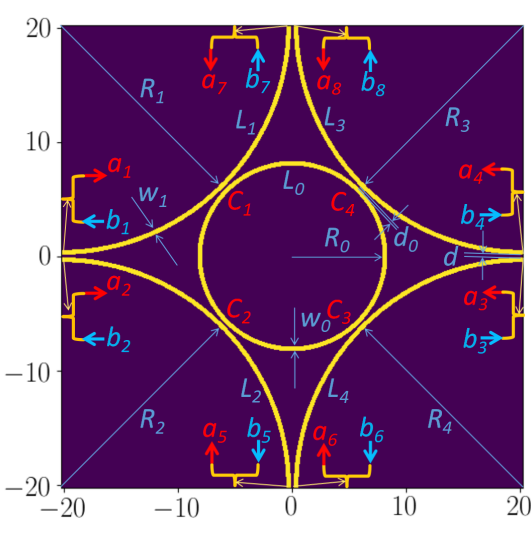

(b) One unit cell.

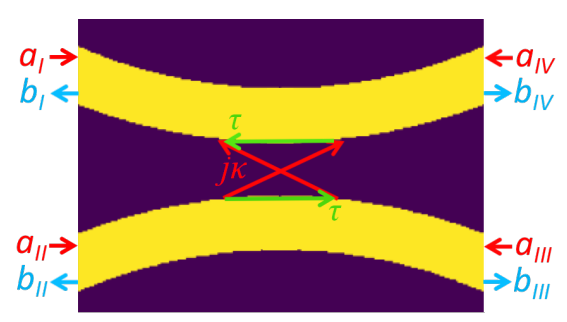

(c) Ports definition of the coupler.

Figure 2: The geometry of the 2D interstitial square coupled MRRs array.

Table 1: $(4 \times 4)$ Transfer Matrices and Scattering Matrix of the Coupler Scattering Matrix Transfer Matrix: $(I, I I)$ Input Ports Transfer Matrix: $(I, I I I)$ Input Ports

$$
\overline{\bar{S}}=\left[\begin{array}{cccc}
0 & 0 & \tau & j \kappa \\
0 & 0 & j \kappa & \tau \\
\tau & j \kappa & 0 & 0 \\
j \kappa & \tau & 0 & 0
\end{array}\right] \quad \overline{\bar{T}}_{(I I I, I V)}^{(I, I I)}=\left[\begin{array}{cccc}
j \kappa & \tau & 0 & 0 \\
\tau & j \kappa & 0 & 0 \\
0 & 0 & -j \kappa & \tau \\
0 & 0 & \tau & -j \kappa
\end{array}\right] \quad \overline{\bar{T}}_{(I I, I V)}^{(I, I I I)}=\left[\begin{array}{cccc}
0 & j \frac{1}{\kappa} & -j \frac{\tau}{\kappa} & 0 \\
j \frac{1}{\kappa} & 0 & 0 & -j \frac{\tau}{\kappa} \\
j \frac{\tau}{\kappa} & 0 & 0 & -j \frac{1}{\kappa} \\
0 & j \frac{\tau}{\kappa} & -j \frac{1}{\kappa} & 0 \kappa
\end{array}\right]
$$

\section{EXAMPLE OF A MICRORING RESONATOR ARRAY}

To demonstrate the procedure presented in Section 3, here we present an example of Miroring Resonators (MRR), i.e., the interstitial square coupled MRR array ${ }^{4}$ that is shown in Fig.2a. The MRR array consists of square MRRs of radius $R_{1}=R_{2}=R_{3}=R_{4}=R$ and interstitial MRR with radius $R_{0}$, as shown in Fig. $2 \mathrm{~b}$ and they are coupled through the 4-port coupler, as shown in Fig. 2c.

\subsection{The Scattering Matrix and Transfer Matrix of the Coupler}

The definition of each port of the $(4 \times 4)$ coupler is shown in Fig. 2c. The incident input waves array and outgoing waves array are given by $\bar{a} \equiv\left[a_{I}, a_{I I}, a_{I I I}, a_{I V}\right]^{T}$ and $\bar{b} \equiv\left[b_{I}, b_{I I}, b_{I I I}, b_{I V}\right]^{T}$ respectively.

$$
\bar{b}=\overline{\bar{S}}^{(4 \times 4)} \bar{a}: \quad \overline{\bar{S}}^{(4 \times 4)}=\left[\begin{array}{cccc}
0 & 0 & \tau & j \kappa \\
0 & 0 & j \kappa & \tau \\
\tau & j \kappa & 0 & 0 \\
j \kappa & \tau & 0 & 0
\end{array}\right]
$$

Depending on the input ports and output ports, the transfer matrix can take different forms. The transfer matrix can be obtained by re-arranging the input/output waves in the scattering matrix given in Eq. (23). The following two transfer matrices are used for the 2D interstitial square coupled MRRs array.

1. $(I, I I)$ Input ports/(III,IV) Output ports:

$$
\bar{o}_{(I I I, I V)}=\overline{\bar{T}}_{(I I I, I V)}^{(I, I I)} \bar{i}_{(I, I I)}
$$

where $\bar{i}_{(I, I I)}=\left[a_{I}, a_{I I}, b_{I}, b_{I I}\right]^{T}$ and $\bar{o}_{(I I I, I V)}=\left[a_{I I I}, a_{I V}, b_{I I I}, b_{I V}\right]^{T}$; and the transfer matrix $\overline{\bar{T}}_{(I I I, I V)}^{(I, I)}$ is given in Table 1. 
2. $(I, I I I)$ Input ports/(II, IV) Output ports:

$$
\bar{o}_{(I I, I V)}=\overline{\bar{T}}_{(I I, I V)}^{(I, I I I)} \bar{i}_{(I, I I I)},
$$

where $\bar{i}_{(I, I I I)}=\left[a_{I}, a_{I I I}, b_{I}, b_{I I I}\right]^{T}$ and $\bar{o}_{(I I, I V)}=\left[a_{I I}, a_{I V}, b_{I I}, b_{I V}\right]^{T}$; and the transfer matrix $\overline{\bar{T}}_{(I I, I V)}^{(I, I I)}$ is given in Table 1.

\subsection{The Constraint-free Port Vectors and the Transfer Matrix}

To obtain the unit-cell transfer matrix $\overline{\bar{T}}_{\text {cell }}$ for the $2 \mathrm{D}$ interstitial square coupled MRRs array, the constraint-free port vectors are determined first as follows: the forward and backward propagating waves at each port of the unit cell have been defined in Fig. 2b: $a_{i}, i=1,2, \cdots, 8$, i.e., the forward propagating waves and $b_{i}, i=1,2, \cdots, 8$ are the backward propagating waves at each port respectively. The incident input port vector is then given as $\bar{i}=\left[\mathbf{a}_{1}, \mathbf{a}_{2}, \mathbf{a}_{5}, \mathbf{a}_{6}, \mathbf{b}_{1}, \mathbf{b}_{2}, \mathbf{b}_{5}, \mathbf{b}_{6}\right]^{T}$. Similarly, the output port vector is given by $\bar{o}=\left[\mathbf{b}_{3}, \mathbf{b}_{4}, \mathbf{b}_{7}, \mathbf{b}_{8}, \mathbf{a}_{3}, \mathbf{a}_{4}, \mathbf{a}_{7}, \mathbf{a}_{8}\right]^{T}$. So the unit-cell transfer matrix is a 8 -by- 8 matrix that connects the input and output port vectors of the unit cell,

$$
\bar{o}=\overline{\bar{T}}_{\text {cell }} \bar{i}
$$

and the transfer matrix can be obtained by connecting all coupling ports with the $(4 \times 4)$ transfer matrices given in Table 1 as follows: first, the $(4 \times 4)$ transfer matrices with various inputs combination can be derived from the $(4 \times 4)$ scattering matrix of the coupler; then the $(8 \times 8)$ transfer matrix $\overline{\bar{T}}_{\text {cell }}^{(8 \times 8)}$ in Eq. (31) can be obtained by connecting all the input ports to all the output ports through the two $(4 \times 4)$ transfer matrices $\overline{\bar{T}}_{(I I I, I V)}^{(I, I I)}$ and $\overline{\bar{T}}_{(I I, I V)}^{(I, I I)}$ given in Eq. (24) and Eq. (25) respectively. Here, ports $(1,2,5,6)$ are used as the input ports with the input waves defined as $i_{1256} \equiv\left[a_{1}, a_{2}, a_{5}, a_{6}, b_{1}, b_{2}, b_{5}, b_{6}\right]^{T}$ and ports $(3,4,7,8)$ are used as the output ports with the output waves defined as $i_{3478} \equiv\left[a_{3}, a_{4}, a_{7}, a_{8}, b_{3}, b_{4}, b_{7}, b_{8}\right]^{T}$, Finally, $\overline{\bar{T}}_{(I I, I V)}^{(I, I I I)}$ is used for couplers $C_{2}$ and $C_{4}$ and $\overline{\bar{T}}_{(I I I, I V)}^{(I, I I)}$ is used for all other couplers to connect all input ports $(1,2,5,6)$ to all output ports $(3,4,7,8)$.

\subsection{Bloch-Floquent Boundary Condition Constraint}

For periodic structure, Floquet-Bloch theory applies and states that the field on the periodic lattice repeats itself with a propagation phase proportional to the propagation wave vector. Mathematically, for any angular frequency $\omega$, a wave vector $\mathbf{k}(\omega)=k_{x}(\omega) \hat{x}+k_{y}(\omega) \hat{y}$ exists such that, ${ }^{16}$

$$
\mathbf{E}\left[i_{x}^{\prime} p_{x}, i_{y}^{\prime} p_{y}\right]=P\left\{\mathbf{k}(\omega), \Delta i_{x}, \Delta i_{y}\right\} \mathbf{E}\left[m p_{x}, n p_{y}\right],
$$

with the propagation phase defined as,

$$
P\left\{\mathbf{k}(\omega), \Delta i_{x}, \Delta i_{y}\right\} \equiv e^{-j\left(k_{x} \Delta i_{x} p_{x}+k_{y} \Delta i_{y} p_{y}\right)},
$$

where $\mathbf{E}$ is the field vector at each port defined on the edges of the unit cell in Fig. $(2 \mathrm{~b}) ; p_{x}, p_{y}$ are the lattice periods in $\hat{x}$ and $\hat{y}$ directions respectively; $\left(i_{x}, i_{y}\right)$ and $\left(i_{x}^{\prime}, i_{y}^{\prime}\right)$ are integers at two different lattice sites and $\left(\Delta i_{x}=i_{x}^{\prime}-i_{x}, \Delta i_{y}=i_{y}^{\prime}-i_{y}\right)$ is the vector difference between them. Note that due to periodicity, $k_{x / y}+$ $M \pi / p_{x / y}, M=(-\infty, \infty)$ is considered to be equivalent. So, it is only necessary to consider $\left(k_{x}, k_{y}\right)$ in the first Brillouin Zone (BZ). Also, for the rest of this paper, we assume that the periods are the same for both $\hat{x}$ and $\hat{y}$ directions: $p_{x}=p_{y}=p$.

It is well known that the transfer matrix connects the field at adjacent unit cells ${ }^{17}$ as follows,

$$
\mathbf{E}\left[\left(i_{x}+1\right) p,\left(i_{y}+1\right) p\right]=\overline{\bar{T}} \mathbf{E}[m p, n p] .
$$


Substituting Eq. (28) into Eq. (27), the following dispersion relation between the angular frequency $\omega$ and the wave vector $\mathbf{k}(\omega)$ as follows,

$$
\left\{\overline{\bar{T}}(\omega)-\overline{\bar{P}}\left\{\mathbf{k}(\omega), \Delta i_{x}, \Delta i_{y}\right\}\right\} \mathbf{E}[m p, n p]=0,
$$

where $\overline{\bar{P}}$ is the phase propagation matrix depending on the wave vector $\mathbf{k}$ and the detailed arrangement of the field vector $\mathbf{E}$. The $\mathbf{k}-\omega$ dispersion curve is then obtained by solving the eigen-values/eigen-vectors problem of Eq. (29) after setting $\Delta i_{x}=\Delta i_{y}=1$ for the unit cell, which requires that the following secular equation to be satisfied,

$$
\left[\overline{\bar{T}}_{\text {cell }}(\omega)-\overline{\bar{P}}_{\text {cell }}\{\mathbf{k}(\omega)\}\right] \bar{i}=0,
$$

with $\bar{i}$ being the input array of $\mathbf{E}$ arranged in an appropriate sequence according to a specific geometry under study.

\subsection{Effective Hamiltonian of the 2D Interstitial Square Array}

Then the effective Hamiltonian secular equation for the obtained $(8 \times 8)$ transfer matrix is obtained according to Eq. (30),

$$
\left\{\overline{\bar{T}}_{\text {cell }}(\omega)-\left[\begin{array}{cc}
\overline{\bar{P}}_{x, \text { cell }}^{(4 \times 4)}\left\{k_{x}(\omega)\right\} & 0 \\
0 & \overline{\bar{P}}_{y, \text { cell }}^{(4 \times 4)}\left\{k_{y}(\omega)\right\}
\end{array}\right]\right\} \bar{i}=0
$$

with

$$
\overline{\bar{P}}_{u, \text { cell }}^{(4 \times 4)}\left\{k_{u}(\omega)\right\}=e^{-j\left(k_{u}(\omega) p_{u}\right)} \overline{\bar{I}}^{(4 \times 4)}, \quad u=x, y .
$$

The eigen wave vector $\mathbf{k}(\omega)=\left[k_{x}(\omega), k_{y}(\omega)\right]^{T}$ can be obtained by solving the determinant equation of Eq. $(22)$,

$$
\left|\overline{\bar{T}}_{\text {cell }}(\omega)-\left[\begin{array}{cc}
\overline{\bar{P}}_{x, \text { cell }}^{(4 \times 4)}\left\{k_{x}(\omega)\right\} & 0 \\
0 & \overline{\bar{P}}_{y, \text { cell }}^{(4 \times 4)}\left\{k_{y}(\omega)\right\}
\end{array}\right]\right|=0 .
$$

In order for the eigen problem of the secular equation in Eq. (30) to have solution, its determinant should be zero according to Eq. (22),

$$
\operatorname{Det} \equiv\left|\overline{\bar{T}}_{c e l l}(\omega)-\overline{\bar{P}}_{c e l l}\{\mathbf{k}(\omega)\}\right|=0,
$$

from which the analytical dispersion relation can be obtained. ${ }^{4}$

\subsection{Simulation Result}

Numerical simulation has been performed to obtain the dispersion curves and eigen modes for the 2D interstitial square coupled MRRs array for a wavelength of $\lambda=1547 \mathrm{~nm}$ with the parameters of $R_{1}=R_{2}=R_{3}=R_{4} \sim$ $10 \mu \mathrm{m} ; \mathrm{w}=w_{0}=500 \mathrm{~nm} ; d=d_{0}=200 \mathrm{~nm} ; R_{0} \sim 4 \mu \mathrm{m}$. Here results are shown for the identical couplers with a $\lambda / 8$ interstitial-ring arc. Finally, without loss of generality, the MRRs have been assumed to be lossless because the loss due to finite radii can be remedied by introducing gain medium through doping.

Fig. 3 shows the dispersion relation of the 2D interstitial square coupled MRRs array: the red dots denote the real part of the wavevector $\Re\left\{k_{x}\right\}$ and the blue dots denote the imaginary part of the wavevector $\Im\left\{k_{x}\right\}$. The zoom-in view for the interstitial-ring arc phase $\Phi(\omega)=[3.74,3.76] \pi$ is shown in Fig. 4.Finally, the field plots of 4 independent eigenmodes are shown in Fig. 5 for the interstitial-ring arc phase of $\Phi(\omega)=3.75 \pi$. 


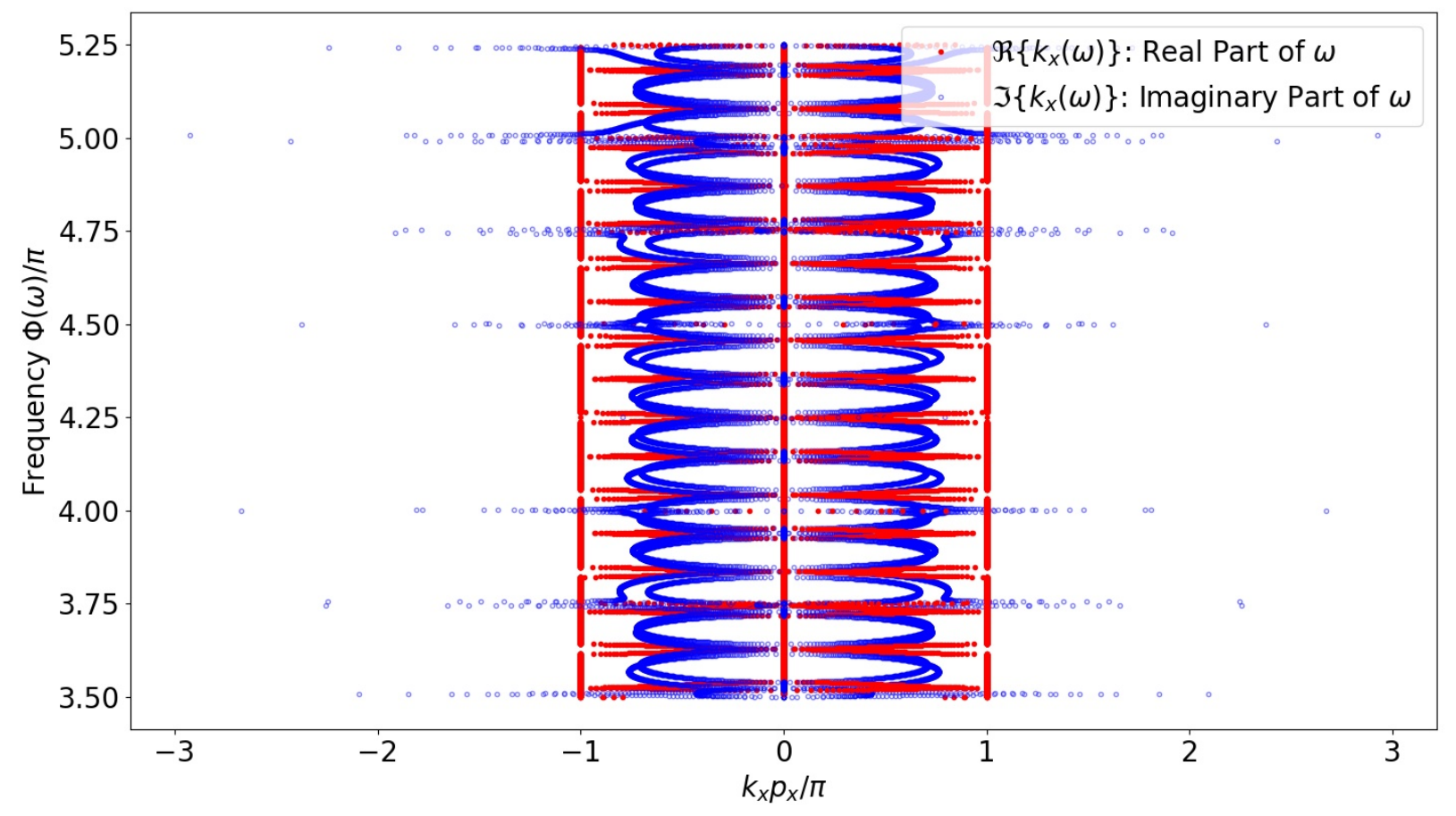

Figure 3: Dispersion relation of the 2D square interstitial MRRS array.

\section{CONCLUSION}

In this paper, an efficient procedure has been presented to compute the effective Hamiltonian of the coupled electromagnetic system. The constraint-free port vectors of all couplers that connect the subsystems and the channels are first chosen according to two rules: 1) channel port vectors that form close loop with the couplers; and 2) port vectors of couplers with most constraints or less freedom. Then the chosen constraint-free port vectors form a system of coupled equations due to the coupling among each other. After that, boundary condition constraints are applied and the effective Hamiltonian is obtained. Finally, the coupled electromagnetic system is solved with the effective Hamiltonian. An example of 2D interstitial square coupled MRRs array has been used to demonstrate the computational recipe and its dispersion relation has been calculated.

\section{ACKNOWLEDGMENTS}

Shaolin Liao is partially supported by start-up grant of SYSU's "100 Top Talents" Program under its "Academic Leaders" Category. Lu Ou is partially supported by the National Natural Science Foundation of China (62002112), Natural Science Foundation of Hunan Province (2021JJ40117), Science and Technology Projects of Changsha City (kq2004027).

\section{REFERENCES}

[1] S. Liao, L. Ou and T. Wong, "Optimal feedback-interferometric fiber laser microphones," Optics Letters, the Optical Society of America (OSA), 45(2): 423-426, 2020. DOI.org/10.1364/OL.384225.

[2] M. Hajizadegan, M. Sakhdari, S. Liao and P.-Y. Chen, "High Sensitivity Wireless Displacement Sensing Enabled by PT-Symmetric Telemetry," IEEE Transactions on Antennas and Propagation, 67(5): 3445-3449, 2019. DOI.org/10.1109/TAP.2019.2905892

[3] Shaolin Liao and Lu Ou, "Rigorous Quantum Formulation of Parity-Time Symmetric Coupled Resonators," Progress In Electromagnetics Research M, 96 : 129-138, 2020. DOI.org/10.2528/PIERM20062602 


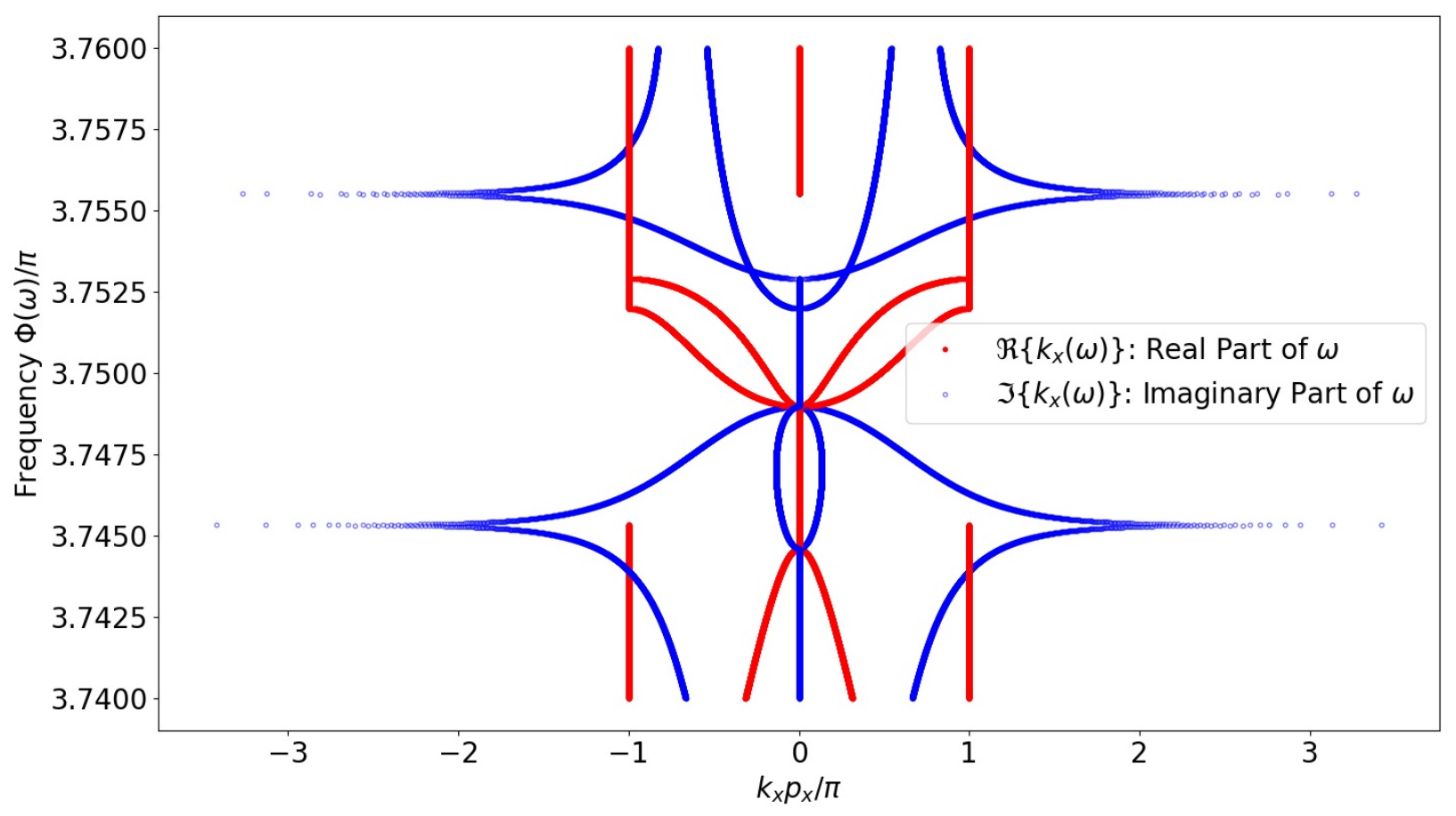

Figure 4: Zoom-in dispersion relation of the 2D square interstitial MRRS array.

[4] S. Liao and L. Ou, "High-Q Interstitial Square Coupled Microring Resonators Arrays," IEEE Journal of Quantum Electronics, 56(4), 2020. DOI.org/10.1109/JQE.2020.2989809

[5] S. Robinson and R. Nakkeeran, "Photonic crystal ring resonator-based add drop filters: a review," Optical Engineering, vol. 52, no. 6, p.060901, Jun. 2013.

[6] Z. Yu, T. Han, G. Wang, G. Qi, F. Luo, and B. Li, " $8 \times 8$ passive noblocking microring resonator crossbar for on-chip WDM-based interconnection network," Optik-International Journal for Light and Electron Optics, vol. 124, no. 18, pp. 3734-3738, Sep. 2013.

[7] U. Dunmeekaew, N. Pornsuwancharoen, and P. P. Yupapin, "New wave-length division multiplexing bands generated by using a Gaussian pulse in a microring resonator system," Microwave and Optical Technology Letters, vol. 52, no. 1, pp. 98-101, 2010.

[8] R. Katti and S. Prince, "Photonic Delay Lines Based on Silicon CoupledResonator Optical Waveguide Structures," Silicon, vol. 10, no. 6, pp.2793-2800, Nov. 2018.

[9] M.-A. Miri and A. Alù, "Exceptional points in optics and photonics," Science, vol. 363, no. 6422, p. eaar7709, Jan. 2019.

[10] X. Zeng, C. M. Gentry, and M. A. Popović, "Four-wave mixing in silicon coupled-cavity resonators with port-selective, orthogonal supermode excitation," Optics Letters, vol. 40, no. 9, p. 2120, May 2015.

[11] K. Guo, L. Yang, X. Shi, X. Liu, Y. Cao, J. Zhang, X. Wang, J. Yang,H. Ou, and Y. Zhao, "Nonclassical Optical Bistability and Resonance-Locked Regime of Photon-Pair Sources Using Silicon Microring Resonator," Physical Review Applied, vol. 11, no. 3, p. 034007, Mar. 2019.

[12] P. M. Alsing and E. E. Hach, "Photon-pair generation in a lossy microring resonator. I. Theory," Physical Review A, vol. 96, no. 3, p.033847, Sep. 2017.

[13] M. Zhang, B. Buscaino, C. Wang, A. Shams-Ansari, C. Reimer, R. Zhu,J. M. Kahn, and M. Lončar, "Broadband electro-optic frequency comb generation in a lithium Niobate microring resonator," Nature, vol. 568,no. 7752, pp. 373-377, Apr. 2019. 

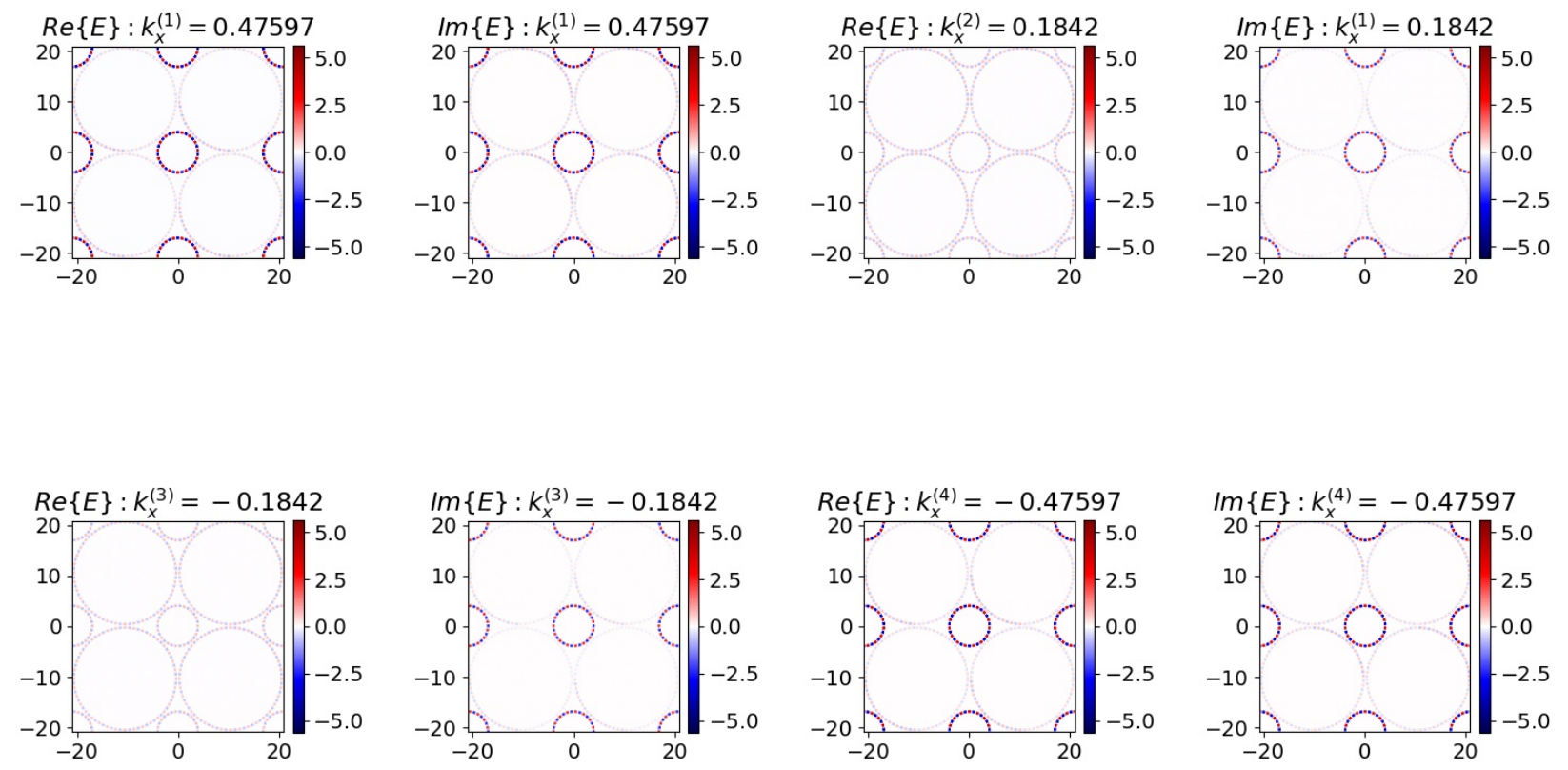

Figure 5: Eigenmode field distribution of the 2D square interstitial MRRS array.

[14] R. E. Scott, P. M. Alsing, A. M. Smith, M. L. Fanto, C. C. Tison,J. Schneeloch, and E. E. Hach, "Scalable controlled-not gate for linear optical quantum computing using microring resonators," Physical Review A, vol. 100, no. 2, p. 022322, Aug. 2019.

[15] S. Wu, Y. Guo, W. Wang, J. Zhou, and Q. Zhang, "Label-free biosensing using a microring resonator integrated with poly-(Dimethyl Siloxane) microfluidic channels," Review of Scientific Instruments, vol. 90, no. 3,p. 035004, Mar. 2019.

[16] J. D. Joannopoulos, ed., Photonic crystals: molding the flow of light, 2nd ed., Princeton University Press, Princeton, 2008.

[17] M. Y. Nada, M. A. K. Othman, and F. Capolino, "Theory of coupled resonator optical waveguides exhibiting high-order exceptional points of degeneracy," Physical Review B, vol. 96, no. 18, p. 184304, Nov. 2017. 